\title{
Growth, Nutrient Utilization and Carcass Characteristics in Broiler Chickens Fed Raw and Alkali Processed Solvent Extracted Karanj (Pongamia glabra) Cake as Partial Protein Supplement
}

\author{
Arun K. Panda, Vadali R.B. Sastry and Asit. B. Mandal \\ Centre of Advance Studies in Animal Nutrition, Indian Veterinary Research Institute Izatnagar, \\ Bareilly, U.P., India, 243122
}

\begin{abstract}
An experiment was conducted to evaluate the effect of dietary incorporation of raw and alkali $[1.5 \% \mathrm{NaOH}$ and $3 \% \mathrm{Ca}$ $(\mathrm{OH})_{2}$, w/w] treated solvent extracted karanj (Pongamia glabra) cake (SKC) on growth performance and carcass characteristics in broiler chickens during 0 to 6 weeks of age. A basal reference diet was formulated containing soybean meal $(\mathrm{SBM})$ as the major protein source. Another six isonitrogenous and isocaloric test diets were formulated incorporating SKC, $1.5 \% \mathrm{NaOH}$ treated SKC $(\mathrm{NaOH}-\mathrm{SKC})$ and $3 \% \mathrm{Ca}(\mathrm{OH})_{2}$ treated SKC $\left(\mathrm{Ca}(\mathrm{OH})_{2}-\mathrm{SKC}\right)$ at 6.43 or $5.5 \%$ during starter phase and 12.86 or $11.0 \%$ during finisher phase replacing SBM nitrogen of reference diet at 12.5 and $25 \%$, respectively. Each diet was offered ad. libitum to 4 replicates of 10 chicks each. The body weight gain and feed efficiency of broilers fed $6.43 \%$ $\mathrm{NaOH}-\mathrm{SKC}$ incorporated diet was comparable with reference diet during 0 to $28 \mathrm{~d}$. However, weight gain and feed efficiency reduced in all karanj cakes incorporated diet during 29 to $42 \mathrm{~d}$. The overall weight gain during 0 to $42 \mathrm{~d}$ was significantly higher in the reference diet. Significantly higher nutrient intake was observed in the reference group except $6.43 \%$ $\mathrm{NaOH}-\mathrm{SKC}$ diet during both the balance trials and $6.43 \% \mathrm{Ca}(\mathrm{OH})_{2}-\mathrm{SKC}$ during $2^{\text {nd }}$ trial. The percent retention of DM, N, $\mathrm{Ca}, \mathrm{P}$ and GE did not differ significantly. Higher liver weight was observed due to dietary incorporation of SKC and $\mathrm{NaOH}-\mathrm{SKC}$ at $25 \%$, and $\mathrm{Ca}(\mathrm{OH})_{2}$-SKC. The gizzard weight was significantly higher in SKC incorporated diet, at both the levels of replacement compared to reference diet. The breast yield lowered significantly when soybean meal nitrogen was replaced with processed or unprocessed karanj cake at $25 \%$ level. The findings suggested that $1.5 \% \mathrm{NaOH}(\mathrm{w} / \mathrm{w})$ treated SKC could be incorporated upto $6.43 \%$ level, replacing $12.5 \%$ of soybean nitrogen of reference diet in broiler chicken upto 4 weeks of age.
\end{abstract}

Key words: Growth performance, carcass characteristics, solvent extracted karanj cake, alkali treatment, broiler chickens

J. Poult. Sci., 45: 199-205, 2008

\section{Introduction}

Escalating cost of feed ingredients and chronic shortage of protein and energy rich animal feeds due to enhanced needs of ever increasing human population are the major threats to reorient poultry industry into an economic enterprise in India. The animal nutritionists are thus compelled to explore the possibilities of feeding non-edible and non-competitive unconventional agro-forest based industrial byproducts, to meet the nutritional requirement of the animals. Since protein sources are costlier, measures are often adapted to partially or completely replace the conventional dietary protein with unconventional protein supplements in order to reduce the cost of production. One such unconventional feed is karanj (Pongamia glabra)

Received: September 26, 2007, Accepted: March 14, 2008

Correspondence: Dr. A.K. Panda, Project Directorate on Poultry, Rajendranagar, Hyderabad, 500030, AP, India.

(E-mail: akpanda59@rediffmail.com) cake, the residue left after oil extraction from karanj seed, is a rich source of protein (28-34\%). In India the availability of karanj seed has been estimated to be $1,300,000$ metric tonne per year (Ministry of Agriculture, 1992).

Though, karanj cake was recommended for its inclusion in different type of poultry ration (Mandal, 1977), it is not commonly used as a feed ingredient for livestock and poultry due to the presence of toxic principle "Karanjin" a furanoflavonoid (Limaye, 1925; Roy et al., 1977). Karanjin is fat-soluble and are removed during the process of oil extraction. However, feeding of de-oiled karanj cake after extraction at higher levels $(>5.0 \%)$ adversely affected the performance (Natnam et al., 1989a, Dhara et al., 1997), which was attributed to the residual toxin (karanjin) still left in the solvent extracted cake. Karanjin can be converted to less toxic intermediates by treatments with alkali (Seshadri and Venkateswarlu, 1943). However, no studies have made for its proper utilization in poultry feeding after detoxification, except physical treatments with water washing and soaking (Mandal and Banerjee, 1974) and 
Table 1. Analyzed chemical composition ( $\%$ ) of feed ingredients

\begin{tabular}{lccccccc}
\hline \hline \multicolumn{1}{c}{ Ingredients } & $\begin{array}{c}\text { ME } \\
(\mathrm{kcal} / \mathrm{kg})\end{array}$ & $\begin{array}{c}\text { Crude } \\
\text { protein }\end{array}$ & $\begin{array}{c}\text { Ether } \\
\text { extract }\end{array}$ & $\begin{array}{c}\text { Crude } \\
\text { fibre }\end{array}$ & $\begin{array}{c}\text { Total } \\
\text { ash }\end{array}$ & Calcium & Phosphorus \\
\hline Maize & 3368 & 9.10 & 3.3 & 2.4 & 1.2 & 0.12 & 0.36 \\
Soybean meal & 2340 & 48.4 & 2.2 & 6.9 & 8.6 & 0.44 & 0.96 \\
De-oiled rice bran & 1800 & 15.1 & 3.2 & 11.8 & 9.4 & 0.36 & 0.71 \\
Solvent extracted karanj cake & 1910 & 33.9 & 4.3 & 5.5 & 4.9 & 0.87 & 0.55 \\
Di-calcium phosphate & - & - & - & - & - & 24.0 & 18.0 \\
Lime stone & - & - & - & - & - & 35.0 & - \\
\hline
\end{tabular}

autoclaving (Natanam et al., 1989b). Recent study by us revealed that chemical treatment of karanj cake with alkali treatments $\left[\mathrm{NaOH}\right.$ and $\left.\mathrm{Ca}(\mathrm{OH})_{2}\right]$ reduced the karanjin content substantially (Panda et al., 2006). The present study was, therefore, conducted to see the effect of dietary incorporation of raw and alkali treated solvent extracted karanj cake on growth performance and carcass characteristics of broiler chickens during 0 to 6 weeks of age.

\section{Materials and Methods}

\section{Processing of Karanj Cake}

Solvent extracted karanj cake (SKC) was procured from local market and was detoxified adopting various physico-chemical methods such as dry heat, water washing, pressure cooking, alkali $\left[\mathrm{NaOH}, \mathrm{Ca}(\mathrm{OH})_{2}\right]$ and acid (HCl) treatments as well as microbiologically with Saccharomyces cerevisiae (strain-49). The residual karanjin left back in the processed cake was quantified through high performance liquid chromatography (HPLC) (Panda et al., 2006). Based on the residual karanjin left back in the processed cake two best methods for detoxification of SKC $\left[1.5 \% \mathrm{NaOH}\right.$ and $\left.3 \% \mathrm{Ca}(\mathrm{OH})_{2}, \mathrm{w} / \mathrm{w}\right]$ were selected. The sodium and calcium hydroxide treated SKC was, thus, prepared by soaking the cake for $24 \mathrm{~h}$ in water $(\mathrm{w} / \mathrm{v}$, $1: 1)$ having sodium hydroxide at $1.5 \%(\mathrm{w} / \mathrm{w})$ and calcium hydroxide at $3 \%(\mathrm{w} / \mathrm{w})$ of cake. The processed cakes were sun dried, ground and packed in gunny bags, in addition to SKC as such, for incorporation into the diet to substitute soybean meal nitrogen partially for broiler feeding.

\section{Stocks and Husbandry}

Two hundred and eighty, day-old commercial broiler (CARIBRO) chicks were wing banded, vaccinated against Marek's disease and distributed in a completely randomized design (CRD) into 7 groups of 40 chicks each, which was further replicated to 4 of 10 chicks each. The chicks of each replicate were housed in the pens of wire floored battery brooders having provision of feeders, waterers and faecal trays from 1 to 42 days of age. The brooder temperature was maintained at $34 \pm 1^{\circ} \mathrm{C}$ up to 7 days of age and gradually reduced to $26 \pm 1^{\circ} \mathrm{C}$ by 21 days of age after which chicks were maintained at room temperature $\left(25^{-}-27^{\circ} \mathrm{C}\right)$. Light was provided continuously throughout the experimental period. Birds were protected against Newcastle disease, infectious bursal disease and fowl pox as routine vaccination schedule. All the chicks were kept under uniform managerial conditions throughout the experimental period.

\section{Experimental Diets}

Feed ingredients were analysed for moisture, crude protein, crude fibre, calcium, phosphorus and ether extract (AOAC, 1990) (Table 1). The apparent metabolizable energy (AME) content of major ingredients were measured by European Reference Method (Bourdillin et al., 1990) except solvent extracted karanj cake, whose AME was measured by practical diet replacement assay (Sibbald et al., 1960) using 6 white leghorn cockerels (24 weeks old) for $3 \mathrm{~d}$, after $7 \mathrm{~d}$ adaptation period. A basal reference diet was prepared containing soybean meal as the principal protein source (D1). Subsequently another 6 test diets were prepared by incorporating SKC (12.5-D2; 25.0-D3), $\mathrm{NaOH}$ treated SKC (12.5-D4; 25.0-D5) and, $\mathrm{Ca}(\mathrm{OH})_{2}$ treated SKC (12.5-D6; 25.0-D7) to replace SBM nitrogen of reference diet at either 12.5 or $25 \%$, respectively. All the diets were kept nearly isonitrogenous and isocaloric through the adjustment of other ingredients and each diet was fed ad lib to 4 replicates of 10 chicks each. The ingredient composition of starter ( $0-4 \mathrm{wk})$ and finisher (5-6 wk), reference and test diets are presented in Table 2 and 3, respectively.

\section{Experimental Procedures}

Individual body weight of chicks and replicate-wise feed intakes were recorded at weekly intervals through out the experimental period. Feed consumption from 1 to $28,29-$ 42 and 1-42 d of age was recorded for each replicate and feed efficiency (weight gain/feed intake) for the respective period was calculated. Mortality was monitored on daily basis.

After 24 and 38 days of experimental feeding, a balance study of 4 days (25-28d; 39-42d) duration each was conducted on all birds to determine the retention of various nutrients by considering a replicate as a single unit. Daily feed offered and faeces voided during this period were recorded. The DM, N, Ca, P and GE in feed and faeces were analyzed as per the AOAC (1990). Based on feed intake and excreta voided, the net retention of DM, $\mathrm{N}, \mathrm{Ca}, \mathrm{P}$ and GE was calculated as retention of nutrients on percent basis.

On $43^{\text {rd }}$ day of experimental feeding, six birds from each dietary group were sacrificed, after removal of feed for two hours with free access to water, through cervical dislocation to observe the weight of dressed carcass, breast, 
Table 2. Ingredient and nutrient composition of starter diets (\%)

\begin{tabular}{|c|c|c|c|c|c|c|c|}
\hline \multirow[b]{2}{*}{ Ingredient } & \multirow{2}{*}{$\frac{\text { Ref }}{\text { D1 }}$} & \multicolumn{2}{|c|}{ SKC } & \multicolumn{2}{|c|}{$\mathrm{NaOH}$ treated $\mathrm{SKC}$} & \multicolumn{2}{|c|}{$\mathrm{Ca}(\mathrm{OH})_{2}$ treated SKC } \\
\hline & & $\begin{array}{c}\mathrm{D} 2 \\
12.5 \%\end{array}$ & $\begin{array}{c}\text { D3 } \\
25 \%\end{array}$ & $\begin{array}{c}\text { D4 } \\
12.5 \%\end{array}$ & $\begin{array}{c}\text { D5 } \\
25 \%\end{array}$ & $\begin{array}{c}\text { D6 } \\
12.5 \%\end{array}$ & $\begin{array}{c}\text { D7 } \\
25 \%\end{array}$ \\
\hline Yellow maize & 60.50 & 59.02 & 55.77 & 59.02 & 55.77 & 59.12 & 56.47 \\
\hline Soybean meal & 35.0 & 30.4 & 26.5 & 30.4 & 26.5 & 30.5 & 26.6 \\
\hline Solvent extracted karanj cake & - & 6.43 & 12.86 & 6.43 & 12.86 & 6.43 & 12.86 \\
\hline De-oiled rice bran & 0.72 & - & - & - & - & - & - \\
\hline Vegetable oil & - & 0.5 & 1.20 & 0.5 & 1.20 & 0.5 & 1.20 \\
\hline Limestone & 1.6 & 1.5 & 1.35 & 1.5 & 1.35 & 1.2 & 0.75 \\
\hline Di-calcium Phosphate & 1.3 & 1.3 & 1.3 & 1.3 & 1.3 & 1.3 & 1.3 \\
\hline Lysine & - & 0.07 & 0.13 & 0.07 & 0.13 & 0.07 & 0.13 \\
\hline Methionine & 0.16 & 0.16 & 0.17 & 0.16 & 0.17 & 0.16 & 0.17 \\
\hline Common salt & 0.4 & 0.4 & 0.4 & 0.4 & 0.4 & 0.4 & 0.4 \\
\hline Trace mineral $^{1}$ & 0.1 & 0.1 & 0.1 & 0.1 & 0.1 & 0.1 & 0.1 \\
\hline \multirow[t]{2}{*}{ Vitamin premix ${ }^{2}$} & 0.22 & 0.22 & 0.22 & 0.22 & 0.22 & 0.22 & 0.22 \\
\hline & \multicolumn{7}{|c|}{ Nutrient composition $(\%)$} \\
\hline Metabolizable energy ( kcal/kg) & 2870 & 2857 & 2850 & 2857 & 2850 & 2865 & 2866 \\
\hline Crude protein & 22.04 & 21.72 & 21.70 & 21.77 & 21.78 & 21.79 & 21.81 \\
\hline Lysine & 1.20 & 1.19 & 1.19 & 1.18 & 1.19 & 1.19 & 1.19 \\
\hline Methionine & 0.51 & 0.54 & 0.55 & 0.53 & 0.55 & 0.53 & 0.55 \\
\hline Calcium & 1.01 & 1.01 & 1.01 & 1.01 & 1.01 & 1.01 & 1.00 \\
\hline Non phytate Phosphorus & 0.4 & 0.4 & 0.4 & 0.4 & 0.4 & 0.4 & 0.4 \\
\hline
\end{tabular}

${ }^{1}$ Trace mineral premix contained: Cu- $.25 \mathrm{mg}$, Fe- $80 \mathrm{mg}$, I-1.2 mg, Mn-65 mg and $\mathrm{Zn}-68 \mathrm{mg}$ per $\mathrm{kg}$ diet.

${ }^{2}$ Vitamins premix contained: Calcium pantothenate- $8 \mathrm{mg}$, choline chloride- $1000 \mathrm{mg}$, niacin- $12 \mathrm{mg}$, pyrodoxine hydrochloride- $1.6 \mathrm{mg}$, vitamin A-8250 IU, vitamin $\mathrm{D}_{3}-1200 \mathrm{ICU}$, vitamin-E $8 \mathrm{mg}$, vitamin $\mathrm{K}-10 \mathrm{mg}$ vitamin $\mathrm{B}_{1^{-}}$

$0.8 \mathrm{mg}$, vitamin $\mathrm{B}_{2}-5 \mathrm{mg}$, vitamin $\mathrm{B}_{12}-8 \mathrm{mcg}$ per $\mathrm{kg}$ diet.

Table 3. Ingredient and nutrient composition of finisher diets $(\%)$

\begin{tabular}{|c|c|c|c|c|c|c|c|}
\hline \multirow[b]{2}{*}{ Ingredient } & \multirow{2}{*}{$\frac{\text { Ref }}{\text { D1 }}$} & \multicolumn{2}{|c|}{ SKC } & \multicolumn{2}{|c|}{$\mathrm{NaOH}$ treated SKC } & \multicolumn{2}{|c|}{$\mathrm{Ca}(\mathrm{OH})_{2}$ treated SKC } \\
\hline & & $\begin{array}{c}\mathrm{D} 2 \\
12.5 \%\end{array}$ & $\begin{array}{c}\text { D3 } \\
25 \%\end{array}$ & $\begin{array}{c}\mathrm{D} 4 \\
12.5 \%\end{array}$ & $\begin{array}{c}\text { D5 } \\
25 \%\end{array}$ & $\begin{array}{c}\text { D6 } \\
12.5 \%\end{array}$ & $\begin{array}{c}\text { D7 } \\
25 \%\end{array}$ \\
\hline Yellow maize & 62.0 & 63.0 & 60.0 & 63.0 & 60.0 & 63.0 & 60.5 \\
\hline Soybean meal & 30.0 & 26.5 & 23.5 & 26.5 & 23.5 & 26.5 & 23.5 \\
\hline Solvent extracted karanj cake & - & 5.5 & 11.0 & 5.5 & 11.0 & 5.5 & 11.0 \\
\hline De-oiled rice bran & 2.8 & 0.0 & 0.0 & 0.00 & 0.16 & 0.45 & 0.26 \\
\hline Vegetable oil & 1.3 & 1.35 & 2.0 & 1.35 & 2.0 & 1.20 & 1.8 \\
\hline Limestone & 1.75 & 1.50 & 1.35 & 1.50 & 1.35 & 1.20 & 0.75 \\
\hline Di-calcium phosphate & 1.3 & 1.3 & 1.3 & 1.3 & 1.3 & 1.3 & 1.3 \\
\hline Lysine & - & - & 0.03 & - & 0.03 & 0.07 & 0.03 \\
\hline Methionine & 0.13 & 0.13 & 0.14 & 0.14 & 0.14 & 0.13 & 0.14 \\
\hline Common salt & 0.4 & 0.4 & 0.4 & 0.4 & 0.4 & 0.4 & 0.4 \\
\hline Trace mineral $^{1}$ & 0.1 & 0.1 & 0.1 & 0.1 & 0.1 & 0.1 & 0.1 \\
\hline \multirow[t]{2}{*}{ Vitamin premix ${ }^{2}$} & 0.22 & 0.22 & 0.22 & 0.22 & 0.22 & 0.22 & 0.22 \\
\hline & \multicolumn{7}{|c|}{ Nutrient composition $(\%)$} \\
\hline Metabolizable energy ( $\mathrm{kcal} / \mathrm{kg})$ & 2948 & 2954 & 2942 & 2954 & 2942 & 2951 & 2946 \\
\hline Crude protein & 20.18 & 20.01 & 20.11 & 20.01 & 20.11 & 20.01 & 20.11 \\
\hline Lysine & 1.04 & 1.01 & 1.00 & 1.01 & 1.00 & 1.01 & 1.00 \\
\hline Methionine & 0.45 & 0.45 & 0.45 & 0.45 & 0.45 & 0.45 & 0.45 \\
\hline Calcium & 1.02 & 1.00 & 0.98 & 1.00 & 0.98 & 0.99 & 0.98 \\
\hline Non phytate Phosphorus & 0.4 & 0.39 & 0.39 & 0.39 & 0.39 & 0.39 & 0.39 \\
\hline
\end{tabular}

${ }^{1}$ Trace mineral premix contained: Cu- $.25 \mathrm{mg}$, Fe- $80 \mathrm{mg}, \mathrm{I}-1.2 \mathrm{mg}$, Mn- $65 \mathrm{mg}$ and $\mathrm{Zn}-68 \mathrm{mg}$ per kg diet.

${ }^{2}$ Vitamins premix contained: Calcium pantothenate- $8 \mathrm{mg}$, choline chloride- $1000 \mathrm{mg}$, niacin- $12 \mathrm{mg}$, pyrodoxine hydrochloride- $1.6 \mathrm{mg}$, vitamin A-8250 IU, vitamin $\mathrm{D}_{3}-1200 \mathrm{ICU}$, vitamin-E $8 \mathrm{mg}$, vitamin $\mathrm{K}-10 \mathrm{mg}$ vitamin $\mathrm{B}_{1^{-}}$ $0.8 \mathrm{mg}$, vitamin $\mathrm{B}_{2}-5 \mathrm{mg}$, vitamin $\mathrm{B}_{12}-8 \mathrm{mcg}$ per $\mathrm{kg}$ diet. 
Table 4. Growth performance of broiler chickens fed karanj cake incorporated diets from 0 to 4 weeks of age

\begin{tabular}{|c|c|c|c|c|c|c|c|c|c|c|}
\hline & & \multicolumn{3}{|c|}{$0-4 \mathrm{wk}$} & \multicolumn{3}{|c|}{$5-6 \mathrm{wk}$} & \multicolumn{3}{|c|}{$0-6 \mathrm{wk}$} \\
\hline \multicolumn{2}{|c|}{ Treatment } & $\begin{array}{c}\text { BWG } \\
(\mathrm{g})\end{array}$ & $\begin{array}{l}\text { FI } \\
(\mathrm{g})\end{array}$ & FE & $\begin{array}{c}\text { BWG } \\
(\mathrm{g})\end{array}$ & $\begin{array}{l}\text { FI } \\
(\mathrm{g})\end{array}$ & FE & $\begin{array}{c}\text { BWG } \\
(\mathrm{g})\end{array}$ & $\begin{array}{l}\text { FI } \\
(\mathrm{g})\end{array}$ & $\mathrm{FE}$ \\
\hline Ref & D1 & $603^{a}$ & $973^{\mathrm{a}}$ & $0.621^{\mathrm{a}}$ & $560^{\mathrm{a}}$ & $1099^{a}$ & $0.510^{\mathrm{a}}$ & $1162^{\mathrm{a}}$ & $2072^{\mathrm{a}}$ & $0.563^{\mathrm{a}}$ \\
\hline \multirow[t]{2}{*}{ SKC } & D2-(12.5) & $533^{\mathrm{b}}$ & $990^{\mathrm{a}}$ & $0.539^{c}$ & $429^{\mathrm{bc}}$ & $974^{\mathrm{b}}$ & $0.439^{\mathrm{b}}$ & $962^{\mathrm{c}}$ & $1964^{\mathrm{ab}}$ & $0.490^{c}$ \\
\hline & D3-(25.0) & $442^{\mathrm{c}}$ & $811^{\mathrm{c}}$ & $0.545^{\mathrm{c}}$ & $323^{\mathrm{e}}$ & $740^{c}$ & $0.437^{\mathrm{b}}$ & $764^{e}$ & $1551^{\mathrm{d}}$ & $0.494^{\mathrm{c}}$ \\
\hline $1.5 \%$ & D4-(12.5) & $597^{\mathrm{a}}$ & $988^{\mathrm{a}}$ & $0.604^{\mathrm{ab}}$ & $466^{\mathrm{b}}$ & $991^{\mathrm{ab}}$ & $0.471^{\mathrm{ab}}$ & $1063^{\mathrm{b}}$ & $1979^{\mathrm{ab}}$ & $0.538^{\mathrm{a}}$ \\
\hline $\mathrm{NaOH}$ & D5-(25.0) & $468^{\mathrm{c}}$ & $832^{c}$ & $0.565^{\mathrm{bc}}$ & $370^{\mathrm{d}}$ & $823^{c}$ & $0.478^{\mathrm{b}}$ & $839^{d}$ & $1655^{\mathrm{cd}}$ & $0.507^{\mathrm{b}}$ \\
\hline $3.0 \%$ & D6-(12.5) & $571^{\mathrm{b}}$ & $932^{\mathrm{b}}$ & $0.615^{\mathrm{ab}}$ & $384^{\text {cd }}$ & $838^{c}$ & $0.455^{\mathrm{ab}}$ & $958^{\mathrm{c}}$ & $1770^{\mathrm{bc}}$ & $0.549^{\mathrm{a}}$ \\
\hline $\mathrm{Ca}(\mathrm{OH})_{2}$ & D7-(25.0) & $442^{\mathrm{c}}$ & $824^{c}$ & $0.525^{\mathrm{c}}$ & $326^{\mathrm{e}}$ & $721^{\mathrm{c}}$ & $0.416^{\mathrm{b}}$ & $768^{e}$ & $1545^{\mathrm{d}}$ & $0.489^{\mathrm{c}}$ \\
\hline SEM & & 5.8 & 19.2 & 0.006 & 7.8 & 28.3 & 0.008 & 11.87 & 44.98 & 0.007 \\
\hline
\end{tabular}

a, b,c, d,e Means with different superscripts in a column differ significantly $(P<0.05)$.

BWG-body weight gain; FI-feed intake; FE-feed efficiency.

giblet (liver, heart and gizzard), abdominal fat, small intestine (SI) and large intestine (LI). All the carcass traits were expressed on percent live weight basis.

\section{Statistical Analysis}

The data pertaining to various parameters were subjected to one-way analysis of variance as per the methods of Snedecor and Cochran (1989). The means in different treatments were tested for statistical significance using Duncan's multiple range test (Duncan, 1955) with significance of $P \leq 0.05$. The body weight data was analysed on individual basis. As the number of observation for the live weight parameter was unequal due to mortality of birds, the live weight change in different dietary treatments were statistically analysed employing the least square analysis of variance technique as per Harvey (1975).

\section{Results}

\section{Body Weight Gain, Feed Intake and Feed Efficiency}

The data on body weight gain, feed intake and feed efficiency of broiler chickens fed reference and test diets during 0 to 4,5 to 6 and 0 to 6 weeks of age are shown in Table 4. Dietary incorporation of SKC or $\mathrm{Ca}(\mathrm{OH})_{2}$ treated SKC at either 6.43 or $12.86 \%$ replacing 12.5 or $25 \%$ replacement of SBM protein of reference diet significantly reduced the body weight gain of broiler chicks during 0 to 4 weeks of age. However, the body weight gain of broiler chicks fed diet incorporated with $6.43 \% \mathrm{NaOH}$ treated $\mathrm{SKC}$ replacing $\mathrm{SBM}$ protein at $12.5 \%$ level was comparable with that of reference diet during the same period. Enhancing the inclusion of $\mathrm{NaOH}$ treated SKC to $12.86 \%$ to replace $25 \%$ protein of SBM significantly depressed the body weight gain. The feed intake of birds fed either SKC or $\mathrm{NaOH}$ treated SKC incorporated diet at $6.43 \%$ level was comparable with that of reference diet during 0 to 4 weeks of age. However, dietary incorporation of $\mathrm{Ca}(\mathrm{OH})_{2}$ treated $\mathrm{SKC}$ at $6.43 \%$ resulted in decrease in feed intake. Dietary incorporation of SKC or $\mathrm{NaOH}$ and $\mathrm{Ca}(\mathrm{OH})_{2}$ treated $\mathrm{SKC}$ at $12.86 \%$ level resulted further decrease in feed intake. The feed efficiency of birds fed either $\mathrm{NaOH}$ and $\mathrm{Ca}(\mathrm{OH})_{2}$ treated $\mathrm{SKC}$ at $6.43 \%$ level was comparable with that of reference diet during 0 to 4 weeks of age. But dietary incorporation of SKC at $6.43 \%$ resulted in significantly poor feed efficiency. Dietary incorporation of $\mathrm{SKC}$ or $\mathrm{NaOH}$ and $\mathrm{Ca}(\mathrm{OH})_{2}$ treated SKC at $12.86 \%$ resulted in poor feed efficiency.

During 29 to 42 days, the body weight gain was significantly lower in the karanj cake diet (with or without processing) at both the level of incorporation (5.5 or $11.0 \%$ ) as compared to that of reference diet. Similar trend in feed intake was observed during the same period except that the feed intake of birds fed $6.43 \% \mathrm{NaOH}$ treated SKC diet was found comparable with that of reference diet. Significantly better-feed efficiency was observed in the birds fed the reference diet. The feed efficiency decreased in all the treated or untreated SKC diet except the $\mathrm{NaOH}$ and $\mathrm{Ca}(\mathrm{OH})_{2}$ treated $\mathrm{SKC}$ at $6.43 \%$ level of incorporation.

The over all body weight gain during 0 to 6 weeks period was significantly higher in the birds fed the reference diet. Though significantly $(P<0.05)$ lowered body weight gain was observed on $\mathrm{NaOH}$ treated SKC containing diet at $6.43 \%$ inclusion, it was significantly higher than on other raw or processed SKC incorporated diets. The feed intake and efficiency during this period followed the same trend as that observed during 0 to 4 weeks of age.

\section{Nutrient Retention}

The data on daily intake and percent retention of DM, $\mathrm{N}, \mathrm{Ca}, \mathrm{P}$ and $\mathrm{GE}$ in birds of different dietary groups during $4^{\text {th }}(25-28 d)$ and $6^{\text {th }}(39-42 d)$ week of experimental feeding have been presented in Table 5 and 6 , respectively. The DM intake (DMI) of the birds fed reference diet was comparable with those of either SKC or $\mathrm{NaOH}$ treated SKC incorporated diet at $6.43 \%$ replacing $12.5 \%$ of SBM protein during 25 to $28 \mathrm{~d}$. However, the DMI decreased in the $\mathrm{Ca}(\mathrm{OH})_{2}$ treated SKC incorporated diet at the same level of incorporation. Significantly lowered DMI were observed when processed or unprocessed SKC was incorporated at $12.86 \%$ replacing $25 \%$ protein of SBM protein. Similar trends were also observed in the percent intake of $\mathrm{N}, \mathrm{Ca}, \mathrm{P}$ and GE. However, the percent retention of DM, N, Ca, P and GE did not differ 
Table 5. Nutrient intake and retention (g or kcal/day/bird) in broiler chickens fed karanj cake incorporated diets during 25 to 28 days of age

\begin{tabular}{llcccccccccc}
\hline \hline \multicolumn{2}{c}{ Treatment } & DMI & DMR & NI & NR & GEI & GER & CaI & CaR & PI & PR \\
\hline Ref & D1 & $60.77^{\mathrm{a}}$ & 66.35 & $13.39^{\mathrm{a}}$ & 41.21 & $249^{\mathrm{a}}$ & 71.19 & $0.638^{\mathrm{a}}$ & 29.86 & $0.419^{\mathrm{a}}$ & 63.88 \\
$\mathrm{SKC}$ & D2-(12.5) & $56.08^{\mathrm{ab}}$ & 64.96 & $12.23^{\mathrm{b}}$ & 42.88 & $232^{\mathrm{a}}$ & 70.50 & $0.589^{\mathrm{ab}}$ & 37.50 & $0.376^{\mathrm{b}}$ & 60.84 \\
& D3-(25.0) & $46.00^{\mathrm{c}}$ & 59.63 & $10.02^{\mathrm{d}}$ & 38.69 & $191^{\mathrm{c}}$ & 64.53 & $0.483^{\mathrm{c}}$ & 37.53 & $0.308^{\mathrm{c}}$ & 53.22 \\
$1.5 \%$ & $\mathrm{D} 4-(12.5)$ & $59.35^{\mathrm{a}}$ & 63.19 & $12.94^{\mathrm{a}}$ & 39.41 & $244^{\mathrm{a}}$ & 66.95 & $0.623^{\mathrm{a}}$ & 32.08 & $0.406^{\mathrm{a}}$ & 50.34 \\
$\mathrm{NaOH}$ & $\mathrm{D}-(25.0)$ & $51.82^{\mathrm{b}}$ & 62.24 & $11.31^{\mathrm{c}}$ & 36.83 & $213^{\mathrm{b}}$ & 66.09 & $0.544^{\mathrm{b}}$ & 28.21 & $0.347^{\mathrm{b}}$ & 53.22 \\
$3.0 \%$ & D6-(12.5) & $52.50^{\mathrm{b}}$ & 65.47 & $11.37^{\mathrm{c}}$ & 34.06 & $218^{\mathrm{b}}$ & 71.75 & $0.551^{\mathrm{b}}$ & 31.05 & $0.362^{\mathrm{b}}$ & 57.87 \\
$\mathrm{Ca}(\mathrm{OH})_{2}$ & D7-(25.0) & $44.81^{\mathrm{c}}$ & 63.78 & $9.76^{\mathrm{d}}$ & 44.88 & $186^{\mathrm{c}}$ & 70.08 & $0.471^{\mathrm{c}}$ & 33.49 & $0.305^{\mathrm{c}}$ & 53.69 \\
$\mathrm{SEM}$ & & 1.37 & 1.06 & 1.40 & 1.71 & 8.02 & 1.02 & 0.02 & 1.29 & 0.009 & 1.45 \\
\hline
\end{tabular}

${ }^{\mathrm{a}, \mathrm{b}, \mathrm{c}, \mathrm{d}}$ Means with different superscripts in a column differ significantly $(P<0.05)$.

DMI-dry matter intake; DMR-dry matter retention; NI-nitrogen intake; NR-nitrogen retention; GEI-gross energy intake; GER-gross energy retention; CaI-calcium intake, CaR-Calcium retention, PI-phosphorous intake, PR -phosphorus retention.

Table 6. Nutrient intake and retention (g or kcal/day/bird) in broiler chickens fed karanj cake incorporated diets during 43 to 47 days of age

\begin{tabular}{llcccccccccc}
\hline \hline \multicolumn{2}{c}{ Treatment } & DMI & DMR & NI & NR & GEI & GER & CaI & CaR & PI & PR \\
\hline Ref & D1 & $94.82^{\mathrm{a}}$ & 74.00 & $19.25^{\mathrm{a}}$ & 65.38 & $387^{\mathrm{a}}$ & 77.63 & $0.956^{\mathrm{a}}$ & $48.29^{\mathrm{a}}$ & $0.654^{\mathrm{a}}$ & 63.44 \\
$\mathrm{SKC}$ & $\mathrm{D} 2-(12.5)$ & $81.71^{\mathrm{b}}$ & 71.85 & $16.45^{\mathrm{b}}$ & 57.61 & $335^{\mathrm{b}}$ & 74.61 & $0.826^{\mathrm{b}}$ & $40.15^{\mathrm{abc}}$ & $0.563^{\mathrm{b}}$ & 69.38 \\
& $\mathrm{D} 3-(25.0)$ & $64.12^{\mathrm{c}}$ & 70.09 & $12.98^{\mathrm{c}}$ & 58.71 & $263^{\mathrm{c}}$ & 73.59 & $0.646^{\mathrm{c}}$ & $32.25^{\mathrm{c}}$ & $0.429^{\mathrm{d}}$ & 60.35 \\
$1.5 \%$ & $\mathrm{D} 4-(12.5)$ & $89.15^{\mathrm{ab}}$ & 71.88 & $17.96^{\mathrm{ab}}$ & 61.71 & $366^{\mathrm{ab}}$ & 76.21 & $0.896^{\mathrm{ab}}$ & $44.06^{\mathrm{ab}}$ & $0.597^{\mathrm{b}}$ & 64.39 \\
$\mathrm{NaOH}$ & $\mathrm{D} 5-(25.0)$ & $78.80^{\mathrm{b}}$ & 68.38 & $16.01^{\mathrm{b}}$ & 58.01 & $323^{\mathrm{b}}$ & 71.54 & $0.796^{\mathrm{b}}$ & $37.08^{\mathrm{bc}}$ & $0.528^{\mathrm{bc}}$ & 60.31 \\
$3.0 \%$ & D6-(12.5) & $86.33^{\mathrm{ab}}$ & 68.81 & $17.48^{\mathrm{ab}}$ & 59.09 & $354^{\mathrm{ab}}$ & 72.61 & $0.873^{\mathrm{ab}}$ & $43.96^{\mathrm{ab}}$ & $0.595^{\mathrm{ab}}$ & 50.38 \\
$\mathrm{Ca}(\mathrm{OH})_{2}$ & D7-(25.0) & $66.77^{\mathrm{c}}$ & 68.44 & $13.56^{\mathrm{c}}$ & 58.15 & $275^{\mathrm{c}}$ & 73.81 & $0.676^{\mathrm{c}}$ & $37.14^{\mathrm{bc}}$ & $0.454^{\mathrm{c}}$ & 57.73 \\
$\mathrm{SEM}$ & & 2.62 & 1.21 & 0.53 & 2.22 & 14.8 & 0.97 & 0.03 & 1.52 & 0.02 & 2.44 \\
\hline
\end{tabular}

${ }^{\mathrm{a}, \mathrm{b}, \mathrm{c}}$ Means with different superscripts in a column differ significantly $(P<0.05)$.

DMI-dry matter intake; DMR-dry matter retention; NI-nitrogen intake; NR-nitrogen retention; GEI-gross energy intake; GER-gross energy retention; CaI-calcium intake, CaR-Calcium retention, PI-phosphorous intake, PR-phosphorus retention.

Table 7. Carcass characteristics (\% live weight) of broiler chickens fed karanj cake incorporated diets

\begin{tabular}{|c|c|c|c|c|c|c|c|c|c|}
\hline \multicolumn{2}{|c|}{ Treatment } & \multirow{2}{*}{$\begin{array}{c}\text { Dressed weight } \\
70.11\end{array}$} & \multirow{2}{*}{$\frac{\text { Liver }}{2.47^{\mathrm{c}}}$} & \multirow{2}{*}{$\begin{array}{c}\text { Heart } \\
0.53\end{array}$} & \multirow{2}{*}{$\frac{\text { Gizzard }}{2.10^{c}}$} & \multirow{2}{*}{$\frac{\text { Abdominal fat }}{0.72}$} & \multirow{2}{*}{$\frac{\text { Small intestine }}{4.77}$} & \multirow{2}{*}{$\frac{\text { Large intestine }}{0.46}$} & \multirow{2}{*}{$\begin{array}{c}\text { Breast weight } \\
15.60^{\mathrm{ab}}\end{array}$} \\
\hline Ref & D1 & & & & & & & & \\
\hline SKC & D2-(12.5) & 72.77 & $3.11^{\mathrm{bc}}$ & 0.50 & $2.55^{\mathrm{ab}}$ & 1.02 & 4.22 & 0.70 & $15.44^{\mathrm{ab}}$ \\
\hline & D3-(25.0) & 70.25 & $3.64^{\mathrm{ab}}$ & 0.44 & $2.91^{\mathrm{a}}$ & 0.72 & 4.43 & 0.57 & $13.91^{\mathrm{bc}}$ \\
\hline $1.5 \%$ & D4-(12.5) & 70.69 & $2.59^{c}$ & 0.52 & $2.29^{\mathrm{bc}}$ & 0.87 & 4.47 & 0.74 & $16.10^{\mathrm{a}}$ \\
\hline $\mathrm{NaOH}$ & D5-(25.0) & 71.38 & $3.75^{\mathrm{a}}$ & 0.50 & $2.32^{\mathrm{bc}}$ & 1.11 & 4.88 & 0.43 & $14.03^{\mathrm{bc}}$ \\
\hline $3.0 \%$ & D6-(12.5) & 70.68 & $3.19^{\mathrm{ab}}$ & 0.48 & $2.33^{\mathrm{bc}}$ & 1.07 & 4.65 & 0.64 & $14.30^{\mathrm{abc}}$ \\
\hline $\mathrm{Ca}(\mathrm{OH})_{2}$ & D7- $(25.0)$ & 70.07 & $3.61^{\mathrm{ab}}$ & 0.50 & $2.43^{\mathrm{bc}}$ & 0.68 & 4.78 & 0.39 & $12.47^{\mathrm{c}}$ \\
\hline SEM & & 0.37 & 0.11 & 0.02 & 0.06 & 0.06 & 0.14 & 0.04 & 0.30 \\
\hline
\end{tabular}

${ }^{\mathrm{a}, \mathrm{b}, \mathrm{c}}$ Means with different superscripts in a column differ significantly $(P<0.05)$.

significantly due to the incorporation of processed or unprocessed SKC at both the levels.

During 39 to $42 \mathrm{~d}$, the DMI of the birds fed reference diet was significantly higher in all the SKC incorporated diets with or without processing except the $\mathrm{NaOH}$ and $\mathrm{Ca}$ $(\mathrm{OH})_{2}$ treated SKC diet at 5.5\% level replacing $12.5 \%$ protein of SBM. The same trend was noticed for the intake of N, GE, Ca and P. The percent retention of all the above nutrients did not differ significantly due to the incorporation of either treated or untreated SKC except that of $\mathrm{Ca}$ retention. Highest and lowest retention of $\mathrm{Ca}$ was observed in the reference and $11.0 \%$ SKC incorporated diet.

\section{Carcass Traits}

Dietary incorporation of raw or processed karanj cake did not affect relative weights (percentage of live body weight) of dressed weight, heart, abdominal fat, SI and LI (Table 7). However, the liver weight increased significantly due to incorporation of SKC and $\mathrm{NaOH}$ treated at $25 \%$ and $\mathrm{Ca}(\mathrm{OH})_{2}$ treated $\mathrm{SKC}$ at both the levels of replacement. The gizzard weight was significantly higher in the SKC incorporated diet at both the levels of replacement 
( 12.5 or $25 \%$ ) as compared to that of reference diet. The breast yield lowered significantly when soybean meal nitrogen was replaced with that of processed or unprocessed karanj cake at $25 \%$ level $(12.86 \%$ during starter and $11.0 \%$ during finisher phase).

\section{Discussion}

The observed poor weight gain and lowered feed intake in the present study on solvent extracted karanj cake (SKC) incorporated diet was in agreement with the findings of Mandal and Banerjee (1974). Mandal and Banerjee (1979) recommended that deoiled karanj cake $(\mathrm{EE}<0.05 \%)$ could safely be included at $5 \%$ in the diet of broiler chicken. Similarly, Dhara et al. (1997) reported that deoiled karanj cake could be included in the diet to a maximum level of $4.45 \mathrm{~kg}$ in $100 \mathrm{~kg}$ of feed replacing $20 \%$ red til cake protein of the reference diet. Higher level resulted in adverse effect on growth, which was attributed to the presence of toxic factors left back in the cake after oil extraction. In the present study, even a lower level of replacement $(12.5 \%)$ of soybean nitrogen with SKC or $\mathrm{Ca}(\mathrm{OH})_{2}$ treated SKC i.e. $6.43 \%$ in the diet resulted in growth depression during 0 to 4 weeks of age. Only dietary replacement of $12.5 \%$ of soybean meal nitrogen with $1.5 \% \mathrm{NaOH}(\mathrm{w} / \mathrm{w})$ treated SKC $(6.43 \%)$ supported the body weight gain up to 4 weeks of age. Higher level of incorporation of the same treated cake $(12.86 \%)$ resulted in growth depression during the same period. However, after $28 \mathrm{~d}$, growth depression was observed in the $\mathrm{NaOH}$ (w/w) treated SKC (5.5\%) incorporated diet as compared to control.

Dietary incorporation of SKC at $6.43 \%$ level depressed the feed efficiency in the present study. However, dietary incorporation of $1.5 \% \mathrm{NaOH}$ or $3 \% \mathrm{Ca}(\mathrm{OH})_{2}$ treated SKC at $6.43 \%$ level alleviated the depression on feed efficiency. Enhancing the inclusion from 6.43 to $12.86 \%$ resulted in lowered feed efficiency. Contrary to the findings of the present study, Natnam et al. (1989 b) reported comparable feed efficiency with reference diet in broiler chicks by incorporating SKC at 10\% level in the diet. Processing of SKC by autoclaving or water soaking did not have any advantage on feed efficiency (Natnam et al 1989b). Dhara et al. (1997) also observed poor FCR by dietary incorporation of $11.20 \%$ to $22.40 \%$ deoiled karanj cake in the diet of Japanese quail. Efficiency of feed utilization for various productive purposes depends on the level of protein and energy (Mellen et al., 1984) and their ratio (Davidson, 1964) in addition to the presence of incriminated factors (Chand, 1987) and the balance of nutrients in the diet (Comb, 1964). Probably the presence of higher levels of residual incriminating factors in diets containing raw and processed SKC at all levels of incorporation except on diet containing $1.5 \% \mathrm{NaOH}$ treated SKC replacing $12.5 \%$ soybean meal protein, resulted in poor FCR in broiler chicks despite feeding of uniformly balanced diets.

Mandal and Banerjee (1982) did not observe any differ- ence on organ weights (liver, heart, kidney and spleen) of cockerels due to dietary replacement of black til cake with deoiled karanj cake at 30\% level. Similarly, Dhara et al. (1997) found no significant variation in weight of different organs (giblet, liver, heart and gizzard) and commercial cuts (neck, wing, thigh, shank, breast and trunk) due to incorporation of deoiled karanj cake upto $22.40 \%$ in the diet of Japanese quail. However, in the present study, dietary inclusion of either SKC or $\mathrm{NaOH}$ treated SKC at $25 \%$ level and $\mathrm{Ca}(\mathrm{OH})_{2}$ treated $\mathrm{SKC}$ at both the levels significantly increased the weight of liver leading to liver hypertrophy. Gizzard weight also increased significantly due to incorporation of SKC at both the levels. The adverse effect of the diet on organ weights relative to the body weight was attributed to the karanjin.

The HPLC analysis revealed that the SKC, $\mathrm{NaOH}$ and $\mathrm{Ca}(\mathrm{OH})_{2}$ treated SKC contained $0.132,0.062$ and $0.082 \%$ karanjin. In the present study the birds during 1 to $28 \mathrm{~d}$ consumed 3.0, 1.4 and $1.8 \mathrm{mg}$ karanjin daily on SKC (84.9 $\mathrm{mg} / \mathrm{kg}), \mathrm{NaOH}(39.9 \mathrm{mg} / \mathrm{kg})$ and $\mathrm{Ca}(\mathrm{OH})_{2}(52.7 \mathrm{mg} / \mathrm{kg})$ treated SKC diet at $6.43 \%$ incorporation level replacing $12.5 \%$ protein of soybean meal. Adverse effect on growth and feed intake was noticed when daily karanjin intake was $1.8 \mathrm{mg}$ and above. However, during 29 to $42 \mathrm{~d}$, birds on average consumed 5.1, 2.4 and $2.7 \mathrm{mg}$ karanjin on the same above diet, respectively. Probably the higher daily karanjin intake $(>1.8 \mathrm{mg}$ ) could be the probable reason for showing adverse effect. Similarly Natnam (1989b) reported the adverse effect of karanj cake feeding is due to the leftover karanjin left over in the processed cake. During 1 to $42 \mathrm{~d}$, the birds on SKC, $\mathrm{NaOH}$ and $\mathrm{Ca}(\mathrm{OH})_{2}$ treated SKC diet at $6.43 \%$ incorporation consumed on an average $3.7,1.7$ and $2.1 \mathrm{mg}$ karanjin daily. In all the diets growth depression was observed during the same period. Assuming $1.8 \mathrm{mg}$ daily intake of karanjin as said, it can be concluded that karanj cake incorporating diet containing more than $39.9 \mathrm{mg} / \mathrm{kg}$ diet is not suitable for broiler chicken feeding.

Thus, the present findings suggested that $1.5 \% \mathrm{NaOH}$ $(\mathrm{w} / \mathrm{w})$ treated SKC could be incorporated up to $6.43 \%$ level, replacing $12.5 \%$ of soybean nitrogen of reference diet of broiler chicken up to 4 weeks of age.

\section{References}

AOAC. Official methods of analysis, Association of Official Analytical Chemists. Washington, D.C. 1990.

Bourdillon A, Carre B, Connan L, Duperray J, Huyghebaert G, Leclerq B, Lessire M, Mcnab J and Wiseman, J. European reference method for the in vivo determination of metabolizable energy with adult cockerels: reproducibility, effect of food intake and comparison with individual laboratory methods. British Poultry Science, 31: 557-565. 1990.

Chand S. Nutritional evaluation of neem seed meal in chicks. Ph. D. Thesis, submitted to the Rohilkhand University, Bareilly. 1987.

Combs GF. Predicting amino acid requirement of chicks based on growth rate, body size and body composition. Feed Processing 23: 46-48. 1964. 
Davidson J. The efficiency of conversion of dietary metabolizable energy into tissue energy in chicken as measured by body analyses. European Association of Animal Production; $3^{\text {rd }}$ symposium on Energy Metabolism, Troon. 1964.

Dhara TK, Chakraborty N, Samanta G and Mandal L. Deoiled karanj ( $P$. glabra vent) cake in the ration of Japanese quail. Indian Journal of Poultry Science, 32: 132-136. 1997.

Duncan DB. Multiple range and F-tests. Biometrics, 11: 1-42. 1955.

Harvey WR. Least square analysis of data with unequal subclass numbers. Agricultural Research Service. United States Department of Agriculture. 1975.

Limaye DB. Karanjin. Part I: A crystalline constituent of the oil from $P$. glabra. Proc. $12^{\text {th }}$ Indian Academy of Science Congress: 118. 1925.

Mandal L and Banerjee GC. Studies on the utilization of karanj (P. glabra) oil cake in Poultry rations. Indian Journal of Poultry Science, 12: 141-147. 1974.

Mandal L. Studies on the utilization of karanj cake - a new feed ingredient in the starters, growers, layers and broiler rations. PhD thesis submitted to Bidhan Chandara Krishi Visshwa Vidyalaya, West Bengal. 1977.

Mandal L and Banerjee GC. Studies on the utilization of karanja (Pongamia glabra vent.) cake in layer diet. Indian Journal of Poultry Science, 14: 105-109. 1979.

Mandal L and Banerjee GC. Studies on the utilization of karanj (P. glabra) cake in poultry rations - Effect on growers and on blood composition and organ weight of cockerels. Indian Veterinary Journal, 59: 385-390. 1982.

Mellen WJ, Hill FW and Dukes HH. Studies on the energy requirement of chickens, 2. Effect and dietary energy level on basal metabolism of growing chickens. Poultry Science, 31: 735-740. 1984.

Ministry of Agriculture. All India final estimates of principal crops. Directorate of Economics of statistics, New Delhi. 1992.

Natanam R, Kadirvel R and Balagopal R. The effect of kernels of karanj ( $P$. glabra vent) on growth and feed efficiency in broiler chicks to 4 weeks of age. Animal Feed Science Technology, 25: 201-206. 1989a.

Natanam R, Kadirvel R and Ravi R The toxic effects of karanj (P. glabra vent) oil and cake on growth and feed efficiency in broiler chicks. Animal Feed Science Technology, 27: 95100. 1989b.

Panda AK, Sastry VRB, Saha, SK and Kumar A. Quantification of karanjin, tannin and trypsin inhibitors in raw and detoxified expeller and solvent extracted karanj (Pongamia glabra) cake. Asian-Australian Journal of Animal Sciences, 12: 1776-1783. 2006.

Roy JHB, Balch CC, Miller EL and Orskov ER. Calculation of the N-requirement for ruminants from nitrogen metabolism studies. In: protein metabolism and nutrition. EAAP: Wangeingen: 126. 1977.

Seshadri TR and Venkateshwarlu V. Synthetic experiments in the Benzo-Pyrone series. Proceedings of the Indian Academy of Sciences, 17(A): 16-19. 1943.

Sibbald IR, Summers JD and Slinger SJ. Determination of metabolizable energy by practical diet replacement method with adult cockerels. Poultry Science, 39: 544. 1960.

Snedecor GW and Cochran WG. Statistical Methods $8^{\text {th }}$ Edn. The Iowa state University Press, Ames, Iowa, USA. 1989. 